\title{
Effects of programmed death-ligand 1 expression on OK-432 immunotherapy following transurethral resection in non-muscle invasive bladder cancer
}

\author{
ZHI-HUA LIU ${ }^{1 *}$, FU-FU ZHENG ${ }^{2 *}$, YU-LING MAO ${ }^{3}$, LIE-FU YE $^{1}$, JUN BIAN $^{4}$, \\ DE-HUI LAI ${ }^{5}$, YUN-LIN YE ${ }^{6}$ and YU-PING DAI ${ }^{2}$ \\ ${ }^{1}$ Department of Urology, Fujian Provincial Hospital, Fuzhou, Fujian 350000; ${ }^{2}$ Department of Urology, \\ First Affiliated Hospital of Sun Yat-sen University; ${ }^{3}$ Affiliated GuangZhou Women and Children's \\ Hospital, Zhongshan School of Medicine, Sun Yat-sen University, Guangzhou, Guangdong 510080; \\ ${ }^{4}$ Department of Urology, Third Affiliated Hospital of Southern Medical University, Guangzhou, Guangdong 510630; \\ ${ }^{5}$ Department of Urology, Fifth Affiliated Hospital of Guangzhou Medical University, Guangzhou, Guangdong 510700; \\ ${ }^{6}$ Department of Urology, Cancer Center of Sun Yat-sen University, Guangzhou, Guangdong 510060, P.R. China
}

Received March 5, 2016; Accepted February 28, 2017

DOI: 10.3892/ol.2017.6080

\begin{abstract}
The present study aimed to investigate the effect of the negative costimulatory molecule programmed death-ligand 1 (PD-L1) on immunotherapy with OK-432, following transurethral resection of bladder tumors in non-muscle invasive bladder cancer (NMIBC), and to elucidate the underlying mechanism. PD-L1 was detected by immunohistochemical staining in tumor specimens from 55 cases of NMIBC following postoperative immunotherapy with OK-432. The PD-L1 mRNA and protein expression levels were measured in the bladder cancer T24 cell line and the human uroepithelial SV-HUC-1 cell line, following treatment with interleukin (IL)-2, interferon (IFN)- $\alpha$ and IFN- $\gamma$, by reverse transcription-quantitative polymerase chain reaction (RT-qPCR) and western blot analysis, respectively. PD-L1 was widely expressed in the NMIBC tumors, with 56.4\% (31/55) of specimens exhibiting positive staining. When compared with PD-L1-negative patients, PD-L1-positive patients exhibited significantly increased recurrence $[48.4 \%(15 / 31)$ vs. $16.7 \%$ $(4 / 24)]$ and progression [16.1\% (5/31) vs. $4.2 \%(1 / 24)]$ rates $(\mathrm{P}<0.05)$. RT-qPCR and western blotting demonstrated that cytokines IL-2, IFN- $\alpha$ and IFN- $\gamma$ markedly upregulated PD-L1 mRNA expression rates and protein levels in bladder
\end{abstract}

Correspondence to: Professor Yu-Ping Dai, Department of Urology, First Affiliated Hospital of Sun Yat-sen University, 58 Zhong Shan street, Guangzhou, Guangdong 510080, P.R. China E-mail: 873592988@qq.com

*Contributed equally

Key words: programmed death-ligand 1, non-muscle invasive bladder cancer, intravesical immunotherapy, OK-432, immune escape cancer T24 cells $(\mathrm{P}<0.05)$, but had no significant effect in non-tumor SV-HUC-1 cells. In conclusion, PD-L1 expression was negatively-associated with the efficacy of OK-432 intravesical immunotherapy in patients with NMIBC. The results indicated that the involved mechanism occurred via upregulation of PD-L1 by immune cytokines, which in turn suppressed the antitumor effectiveness of the immune system, thereby promoting tumor recurrence and progression.

\section{Introduction}

Bladder urothelial tumors are divided into two main groups: Non-muscle invasive bladder cancer (NMIBC) and invasive bladder cancer. NMIBC accounts for $75-80 \%$ of primary diagnoses (1). A transurethral resection of bladder tumor (TURBT) is the preferred treatment for NMIBC, however, almost $70 \%$ of patients with intermediate- or high-risk NMIBC relapse within 6-12 months of surgery, and $\sim 25 \%$ progress to a higher stage disease $(2,3)$. Therefore, postoperative adjuvant intravesical therapy, including intravesical chemotherapy and immunotherapy, is considered necessary for these patients. The probabilities of recurrence and progression may reduce to 42-65\% under appropriate intravesical therapy at 5 years (4). Although carcinoma in situ (CIS) is an NMIBC, it is often poorly differentiated and highly malignant; therefore, the probability of myometrial invasion is significantly increased compared with papillary stage Ta and T1 bladder cancer, and Bacillus Calmette-Guérin (BCG) is the recommended agent for adjuvant intravesical immunotherapy in these cases.

Programmed death-ligand 1 (PD-L1; also termed B7-H1) is a member of the B7 family of costimulatory molecules; it is a cell surface glycoprotein that promotes apoptosis by binding to its surface receptor, programmed cell death-1 (PD-1), in $\mathrm{T}$ cells and $\mathrm{B}$ cells, thereby inhibiting host immune function. PD-L1 has also been implicated in tumor immune escape $(5,6)$.

OK-432 (also termed Picibanil), which is a penicillin-killed and lyophilized preparation of a low-virulence strain of 
Streptococcus pyogenes (group A), has been successfully used as an immunotherapeutic agent against numerous types of malignancies (7-10). It has been reported that OK-432 elicits antitumor effects by stimulating immunocompetent cells, including macrophages, T cells and natural killer (NK) cells, and by inducing helper T-cell 1-type cytokines, including interferon (IFN)- $\gamma$, tumor necrosis factor (TNF)- $\alpha$, and interleukin (IL)-6, IL-8, IL-10, IL-12 and IL-18 $(11,12)$, which may augment cytotoxic $\mathrm{T}$ lymphocytes to antitumor cells.

In the present study, the expression rates of PD-L1 were evaluated in cases of NMIBC patients who had undergone intravesical immunotherapy, and these expression levels were compared with patient outcome. The effects of specific cytokines on PD-L1 expression rates in vitro were also examined, and the potential mechanism of PD-L1 negativity on the efficacy of intravesical immunotherapy in patients with NMIBC was investigated.

\section{Materials and methods}

Patient selection and treatment. Tissue samples were collected from 55 patients ( 43 men and 12 women) with NMIBC who had been treated in our institution (First Affiliated Hospital of Sun Yat-sen University, Guangzhou, China) between January 2010 and January 2012. All the patients had undergone TURBT followed by intravesical immunotherapy with OK-432. None of the patients had received chemotherapy or radiotherapy prior to surgery. The mean age of patients was 55 years (range, 28-77 years), and their clinical features are summarized in Table I. Patients presenting with muscle-invasive transitional cell carcinoma or concurrent malignancy of another organ were excluded. Patients with bladder CIS were also excluded.

The treatment protocol consisted of intravesical immunotherapy (3 KE Picibanil/30 ml saline) administered 12 times over 7 months. Instillations were administered every week for 6 weeks and then every 4 weeks for 6 months. Cystoscopy was performed every 3 months after TURBT, and biopsies were performed if a suspected lesion was found in order to determine whether tumor recurrence had occurred. Time-to-first recurrence, time-to-progression $>$ pT1 stage and toxicity were recorded.

All patients were followed-up for at least 12 months post-surgery. The present study was approved by the Ethics Committee of First Affiliated Hospital of Sun Yat-sen University. Written informed consent was obtained from all patients prior to enrollment in the present study.

Immunohistochemistry. Tissue samples collected from patients were fixed in $10 \%$ dampen formaldehyde solution and embedded in paraffin. In order to detect expression of PD-L1, 5- $\mu \mathrm{m}$ thick histological sections were prepared and incubated with normal goat serum (Wuhan Boster Biological Technology, Ltd., Wuhan, China) for $1 \mathrm{~h}$ at room temperature to block endogenous peroxidase activity. Sections were incubated at $4^{\circ} \mathrm{C}$ overnight with primary antibodies against PD-L1 (dilution, 1:200; catalog no. ab58810; Abcam, Cambridge, UK). Immunohistochemical staining was performed using the SABC kit according to the manufacturer's protocols (Wuhan Boster Biological Technology, Ltd.). Sections were counterstained with hematoxylin and then dehydrated, cleared and mounted.
To evaluate the specificity of the reaction, phosphate-buffered saline (PBS) was used for replacing the primary antibody as the negative control. Subsequent to being washed twice in PBS, the sections were incubated with biotinylated secondary antibody (dilution, 1:500; catalog no. E0466; Dako; Agilent Technologies, Inc., Santa Clara, CA, USA) for $20 \mathrm{~min}$ at room temperature, washed twice in PBS and then incubated with horseradish-peroxidase (HRP)-labeled streptavidin (dilution, 1:2,000; catalog no. K5007; Dako; Agilent Technologies, Inc.) for an additional $20 \mathrm{~min}$. The results were visualized by fluorescence microscopy (Leica Microsystems GmbH, Wetzlar, Germany) following chromogenic staining with diaminobenzidine (Wuhan Boster Biological Technology, Ltd.) and analyzed using Image-Pro Plus software version 6.0 (Media Cybernetics, Inc. Rockville, MD, USA). The percentage of PD-L1-positive tumor cells among the total number of tumor cells was scored in five randomly selected high-power fields (magnification, $\mathrm{x} 400$ ) and percentages $>10 \%$ were classified as PD-L1-positive.

Cell culture. Unless otherwise specified, all reagents were standard laboratory stocks. Human uroepithelial (SV-HUC-1) and bladder cancer (T24) cell lines were purchased from the Cell Bank of the Chinese Academy of Sciences (Shanghai, China). The cells were cultured in Ham's F12 medium and Roswell Park Memorial Institute-1640 media, respectively, supplemented with $10 \%$ fetal bovine serum and $2 \%$ penicillin/streptomycin, and incubated in a humidified atmosphere of $5 \% \mathrm{CO}_{2}$ at $37^{\circ} \mathrm{C}$. Cells at $<80 \%$ confluence were cultured for $24 \mathrm{~h}$ in the presence of IL-2, IFN- $\alpha$, IFN- $\gamma$ (final activity concentration, 1,000 U/ml; Prospec-Tany TechnoGene, Ltd., East Brunswick, NJ, USA) or PBS as a control. The cells were harvested for analysis by RT-qPCR and western blotting.

$R T-q P C R$. Total RNA (500 ng) was extracted from cultured T24 and SV-HUC-1 cells using TRIzol reagent (Invitrogen; Thermo Fisher Scientific, Inc., Waltham, MA, USA) according to the manufacturer's protocol. Reverse transcription was performed using the PrimeScript RT reagent (Perfect Real Time) kit (Takara Biotechnology Co., Ltd., Dalian, China). RT-qPCR was performed using the Roche capillary-based Light Cycler 2.0 system (Roche Diagnostics, Indianapolis, IN, USA) and the SYBR Premix Ex Taq ${ }^{\text {TM }}$ (Perfect Real Time) kit (Takara Biotechnology Co., Ltd.) under standard thermocycling conditions (start at $95^{\circ} \mathrm{C}$ for $15 \mathrm{~min}$ and 40 cycles of $95^{\circ} \mathrm{C}$ for $15 \mathrm{sec}, 55^{\circ} \mathrm{C}$ for $30 \mathrm{sec}$ and $72^{\circ} \mathrm{C}$ for $30 \mathrm{sec}$ ). PD-L1-specific TaqMan probes and primers were purchased from Invitrogen (Thermo Fisher Scientific, Inc.). Appropriate dilutions $(1: 1,000)$ of single strand cDNA were prepared for subsequent PCR using $\beta$-actin as the quantitative control. Primers were as follows: PD-L1 forward, 5'-CACTCATCATTGGCTTTGGTA TTTCAG-3' and reverse, 5'-CGACAGCTCATCTTTGCC TTCTTTG-3'; and $\beta$-actin forward, 5'-AGCGGGAAATCG TGCGTGAC-3' and reverse, 5'-ACTCCTGCTTGCTGATCC ACATC-3'. Each experiment was performed in triplicate and analyzed using the $2^{-\Delta \Delta \mathrm{Cq}}$ method of quantification (13).

Western blot analysis. Cultured SV-HUC-1 and T24 cells were washed three times in PBS and lysed in 1X SDS buffer. Protein concentrations were determined using a Bio-Rad DC 
Table I. Incidence of recurrence and progression in association with PD-L1.

\begin{tabular}{lccc}
\hline $\begin{array}{l}\text { PD-L1 } \\
\text { expression }\end{array}$ & $\begin{array}{c}\text { Patients, } \\
\mathrm{n}\end{array}$ & $\begin{array}{c}\text { Recurrences, } \\
\mathrm{n}(\%)\end{array}$ & $\begin{array}{c}\text { Progression, } \\
\mathrm{n}(\%)\end{array}$ \\
\hline Positive & 31 & $15(48.4)$ & $5(16.1)$ \\
Negative & 24 & $4(16.7)^{\mathrm{a}}$ & $1(4.2)^{\mathrm{a}}$ \\
Total & 55 & $19(34.5)$ & $6(10.9)$ \\
\hline
\end{tabular}

${ }^{\mathrm{a}} \mathrm{P}<0.05$. PD-L1, programmed death-ligand 1.

protein assay kit (Bio-Rad Laboratories, Inc., Hercules, CA, USA) according to the manufacturer's protocol. The proteins were separated by $10 \%$ SDS-PAGE and electrophoretically transferred to Immobilon polyvinylidene difluoride membranes (EMD Millipore, Billerica, MA, USA). Blocking was performed using $10 \%$ non-fat dried milk freshly prepared in Tween-20 tris-buffered saline [0.1\% Tween-20 in $100 \mathrm{mM}$ tris-CL (pH 7.5) and $0.9 \% \mathrm{NaCl}$ ), which was agitated for $15 \mathrm{~min}$ at room temperature overnight. The membranes were incubated with primary antibodies against PD-L1 (dilution, 1:200; catalog no. ab58810; Abcam) and $\beta$-actin (dilution, 1:25; catalog no. SAB5500001; Sigma-Aldrich; Merck KGaA, Darmstadt, Germany) overnight at $4^{\circ} \mathrm{C}$, followed by incubation with their respective HRP-conjugated secondary antibodies $(1: 10,000$; catalog no. NA933, GE Healthcare; NJ, USA) for $2 \mathrm{~h}$ at room temperature. Signals were detected by enhanced chemiluminescence (GE Healthcare Life Sciences, Chalfont, UK) and developed on X-ray film (Fuji Photo Film; FUJIFILM Corporation, Tokyo, Japan). Each experiment was repeated in triplicate.

Statistical analysis. All in vitro experiments were performed in triplicate. Data are presented as the mean \pm standard deviation of three independent repeats. Comparisons between two groups and the association between PD-L1 mRNA expression level or PD-L1 protein expression levels and cytokines were analyzed using SPSS 17.0 (SPSS, Inc., Chicago, IL, USA), GraphPad Prism 5.0 software (GraphPad Software, Inc., La Jolla, CA, USA) and Microsoftexcel(Microsoft Corporation, Redmond, WA, USA). Significance was determined using $\chi^{2}$ test. $\mathrm{P}<0.05$ was considered to indicate a statistically significant difference.

\section{Results}

Association between PD-L1 and efficacy of intravesical immunotherapy. A total of 31/55 (56.4\%) NIMIBC specimens stained positive for PD-L1, with cytoplasmic or membrane staining intensity ranging from light yellow to brown (Fig. 1). No plasma membrane expression of PD-L1 was observed in the normal urothelium adjacent to the malignant urothelium. PD-L1 expression was identified to be significantly associated with pathological grade and histological stage of NMIBC; the percentages of positive PD-L1 immunostaining were $38.1 \%(8 / 21)$ and $67.6 \%$ (23/34) for pTa and pT1 tumors, respectively $(\mathrm{P}<0.05)$. PD-L1-positive patients who had undergone intravesical immunotherapy with OK-432 exhibited
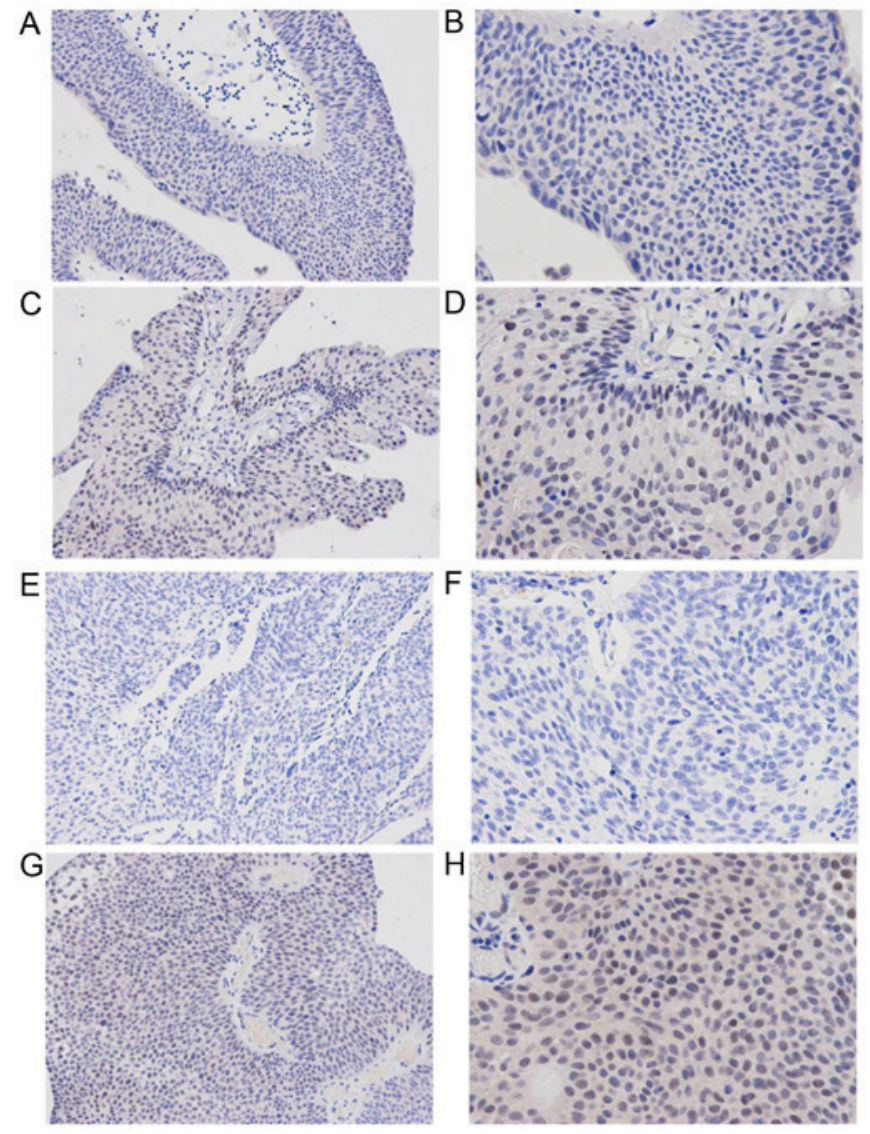

Figure 1. Immunohistochemistry showing levels of PD-L1 expression in cases of NMIBC. (A and B) Absence of PD-L1 staining and (C and D) marked membranous PD-L1 staining in cases of Ta NMIBC. (E and F) Absence of PD-L1 staining and $(\mathrm{G}$ and $\mathrm{H})$ marked membranous PD-L1 staining in cases of T1 NMIBC. (A, C, E and G) Low magnification (x200); (B, D, F and H) High magnification (x400). NMIBC, non-muscle invasive bladder cancer; PD-L1, programmed death-ligand 1.

significantly increased probabilities of recurrence and progression compared with PD-L1-negative patients, with recurrence rates of $48.4 \%(15 / 31)$ and $16.7 \%(4 / 24)$, respectively, and progression rates of $16.1 \%(5 / 31)$ and $4.2 \%(1 / 24)$, respectively $(\mathrm{P}<0.05$; Table I). PD-L1 expression was not associated with patient sex, and there was no association with patient age or tumor size (Table II).

Upregulation of PD-L1 mRNA in T24 bladder cancer cell lines. RT-qPCR results demonstrated that relative expression rates of PD-L1 mRNA were significantly increased in T24 cells treated with IL-2, IFN- $\alpha$ and IFN- $\gamma(1.22 \pm 0.16,1.37 \pm 0.21$ and $2.45 \pm 0.19$, respectively) compared with untreated T24 cells $(1.00 \pm 0.08 ; \mathrm{P}<0.05)$. By contrast, no significant differences were observed between treated and untreated uroepithelial SV-HUC-1 cells (Fig. 2). These results indicated that the cytokines IL-2, IFN- $\alpha$ and IFN- $\gamma$ upregulated PD-L1 mRNA expression in T24 cell lines, but had no significant effect in SV-HUC-1 cell lines.

Upregulation of PD-L1 protein in T24 cell lines. Western blot analysis demonstrated that the levels of PD-L1 protein were increased in T2 4 cells treated with IL-2, IFN- $\alpha$ and IFN- $\gamma$ $(2.1 \pm 0.41,2.77 \pm 0.29$ and $3.45 \pm 0.39$, respectively) compared 
Table II. Clinical characteristics of patients in association with PD-L1 expression status.

\begin{tabular}{|c|c|c|c|}
\hline Age, years (mean \pm SD) & $\begin{array}{l}\text { PD-L1-positive } \\
61 \pm 10\end{array}$ & $\begin{array}{c}\text { PD-L1-negative } \\
64 \pm 11\end{array}$ & $\begin{array}{c}\chi^{2} \text { analysis } \\
\text { n.s. }\end{array}$ \\
\hline Sex, $n$ & & & n.s. \\
\hline Male & 24 & 19 & \\
\hline Female & 7 & 5 & \\
\hline Size of tumors, $n$ & & & n.s. \\
\hline$<3 \mathrm{~cm}$ & 11 & 7 & \\
\hline$\geq 3 \mathrm{~cm}$ & 20 & 14 & \\
\hline Histological grade, $\mathrm{n}$ & & & $\mathrm{P}<0.005$ \\
\hline High & 15 & 7 & \\
\hline Low & 9 & 24 & \\
\hline Pathological grade, $\mathrm{n}$ & & & $\mathrm{P}<0.005$ \\
\hline $\mathrm{pTa}$ & 8 & 13 & \\
\hline pT1 & 23 & 11 & \\
\hline
\end{tabular}

n.s., not significant; SD, standard deviation; PD-L1, programmed death-ligand 1.
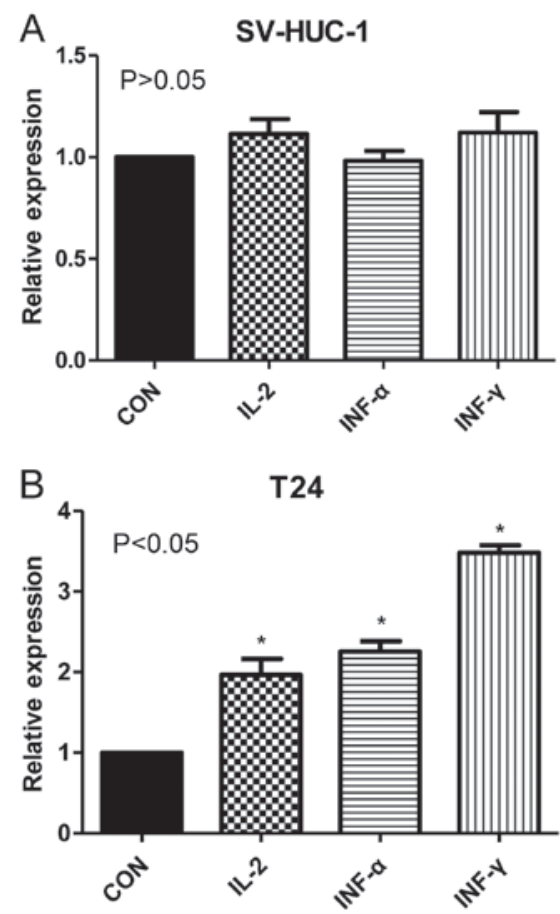

Figure 2. Programmed death-ligand 1 mRNA levels were determined by reverse transcription polymerase chain reaction in (A) SV-HUC-1 and (B) T24 cell lines following treatment with cytokines IL-2, IFN- $\alpha$ and IFN- $\gamma$. Cells that were not treated with cytokines were used as a control. ${ }^{*} \mathrm{P}<0.05$ vs. control. CON, control; IL-2, interleukin-2; INF- $\alpha$, interferon- $\alpha$; INF- $\gamma$, interferon- $\gamma$.

with untreated cells $(1.00 \pm 0.08)(\mathrm{P}<0.05)$. By contrast, the corresponding levels in SV-HUC-1 cells compared with untreated cells $(0.21 \pm 0.08)$ were $0.27 \pm 0.05,0.44 \pm 0.11$ and $0.37 \pm 0.19$, respectively $(\mathrm{P}>0.05)$. These findings indicated that the cytokines IL- 2 , IFN- $\alpha$ and IFN- $\gamma$ increased expression of PD-L1 protein in bladder cancer T2 4 cell lines, but had no significant effect in non-tumor SV-HUC-1 cell lines (Fig. 3).

\section{Discussion}

The majority of patients with NMIBC $(>70 \%)$ relapse or progress to a higher level of invasive bladder cancer, as reflected by increases in the numbers, sizes, stages and grades of the tumors, as well as the frequency of recurrence and the presence of CIS (14). Therefore, postoperative adjuvant intravesical immunotherapy may be recommended in intermediate- and high-risk patients with NMIBC $(3,14)$. Tumor immune escape mechanisms may also be important in recurrence and progression. Immunotherapeutic agents act by inducing a local immune response to prevent tumor recurrence (15). BCG has become the most commonly used intravesical agent and is known to be superior to other intravesical agents for the prevention of tumor recurrence $(16,17)$. However, the clinical applications of BCG have been limited by adverse side effects and difficulties in its preparation and preservation.

OK-432 (also known as Sapylin) is a biological reaction modifier derived from the managed and acquired Su-strains (toxic mutant strain) of human group A hemolytic Streptococcus. Clinical trials have demonstrated that OK-432 may be effective in the treatment of malignant pleural effusion and ascites, in solid tumors, including those from lung, stomach, liver and breast cancer, and in lymphatic cancer, such as metastases (18). OK-432 has also been shown to improve and prolong patient survival, alleviate systemic conditions (particularly those involving the immune function) and reduce the side effects of chemotherapy and radiotherapy (19-21). In our center (Department of Urology, First Affiliated Hospital of Sun Yat-sen University), good results have been achieved in preventing recurrence and progression of NMIBC by administering OK-432 as an intravesical immunotherapeutic agent (22). Previous studies have proposed that the primary antitumor role of OK-432 in bladder carcinoma involves mobilization of the cellular immune system by activating macrophages, NK cells, lymphokine-activated killer cells and various $\mathrm{T}$ cells $(23,24)$. Secondary roles include increasing 
A

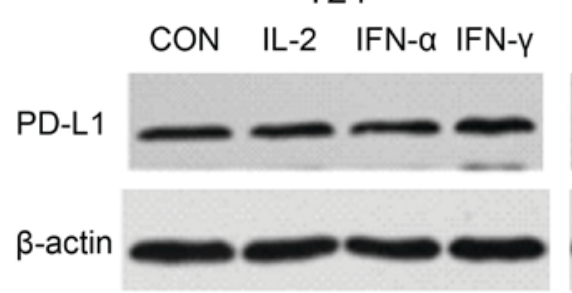

B

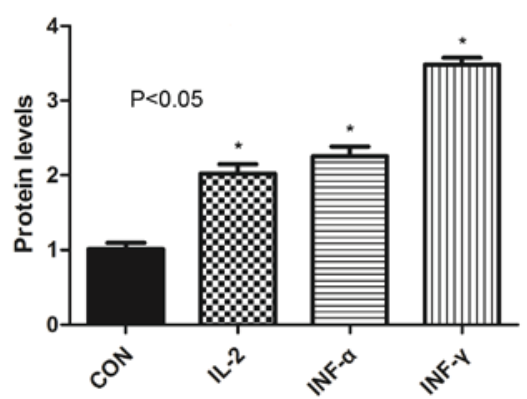

SV-HUC-1
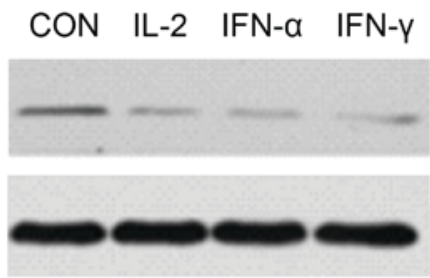

SV-HUC-1

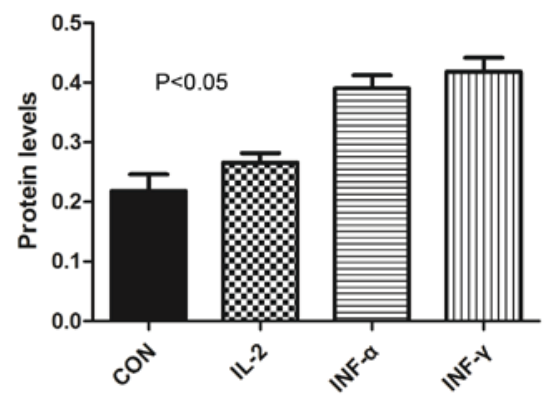

Figure 3. (A) PD-L1 protein level in T24 and SV-HUC-1 cell lines following treatments with cytokines IL-2, IFN- $\alpha$ and IFN- $\gamma$, as detected by western blotting using anti-PD-L1 and anti- $\beta$-actin antibodies. Cells that were not treated with cytokines were used as a control. (B) The quantitation of the expression protein levels. ${ }^{*} \mathrm{P}<0.05$ vs. control. CON, control; IL-2, interleukin-2; INF- $\alpha$, interferon- $\alpha$; INF- $\gamma$, interferon- $\gamma$; PD-L1, programmed death-ligand 1.

the binding capacity of these cells to the tumor cells, thereby enhancing the antitumor effect (25). OK-432 not only acts in a similar manner to BCG in stimulating peripheral blood mononuclear cells to produce cytokines (including TNF, INF and IL-2, which also perform roles in antitumor mechanisms), but has been identified to be significantly stronger than BCG in activating NK cells and inducing the secretion of IL-2, TNF and INF (26).

In the present study, 55 patients with NMIBC were divided into 2 groups according to their tumor expression levels of PD-L1. No significant differences were observed between the 2 groups with respect to patient age, sex, tumor size and grade. However, OK-432 was revealed to be more effective in preventing recurrence and progression in patients in the PD-L1-negative group compared with those in the PD-L1-positive group and historical controls. Constitutive expression of PD-L1 is usually confined to macrophage lineage cells $(27,28)$, however, abnormal expression of PD-L1 has been reported in a variety of human tumors, including breast, ovarian, lung, colon and renal cancer, lymphoma and melanoma (29-32). In the present study, we hypothesized that tumor cells in NMIBC bind to T-cell surface receptor PD-1, or a putative non-PD-1 receptor, via PD-L1, to promote antigen-specific T-cell apoptosis or dysfunction. This may enable the cells to escape immune-mediated antitumor effects by inhibiting the immune response. Such interactions between malignant cells and the immune system serve an important role in tumor formation and development. Furthermore, as T cells are considered essential for acquired antitumor immunity, it was concluded that PD-L1 may also serve an important role in the recurrence and progression of NMIBC. These hypotheses were supported by observations in the literature. NMIBC is one of the most responsive human malignancies to immunotherapy, and host immunity appears to modulate bladder cancer pathogenesis (15). Furthermore, patients with bladder cancer may manifest acquired immune dysfunction, which appears to affect lymphocytes and is associated with tumor stage (33-35). It has become apparent that T-cell-inhibitory PD-L1 is aberrantly expressed in numerous types of cancer and has been implicated as a mechanism for tumor cells to evade the host immune system $(36,37)$. Together, these previous findings indicated that abnormal expression of PD-L1 in bladder cancer may contribute to the ability of the tumor to resist host antitumor immune cells, thereby reducing the benefit of intravesical immunotherapy and promoting tumor recurrence and progression. As such, PD-L1-positive patients exhibit poorer responses to treatment with OK-432 compared with PD-L1-negative patients. Consistent with these findings, the present immunohistochemistry observations revealed that PD-L1 expression was increased in recurrent cases that had been treated with OK-432 compared with non-recurrent cases.

The RT-qPCR results indicated that the cytokines IL-2, IFN- $\alpha$ and IFN- $\gamma$ were associated with increased expression of PD-L1 at the mRNA and protein levels in T24 bladder cancer cell lines in vitro. This effect was not observed in normal uroepithelial SV-HUC-1 cell lines. IFN- $\gamma$ may be involved in the regulation of PD-L1 via certain signaling pathways, including the Janus kinase (JAK)/signal transducer and activator of transcription 1 (STAT1)/interferon regulatory factor-1 (IRF-1); phosphoinositide-3 kinase (PI3K)/Akt/mechanistic target of rapamycin (mTOR)/S6 K and methyl ethyl ketone (MEK)/extracellular signal-regulated kinase (ERK)/STAT1 pathways (38). Previous studies have confirmed that the JAK/STATI/IRF-1 and MEK/ERK/STAT1 signaling pathways are implicated in the association between IFN $-\gamma$ and PD-L1 (39). In addition, the deletion or mutation of the phosphatase and tension homolog gene may contribute to an increase in PD-L1 expression via the PI3K/Akt/mTOR/S6K signaling pathway (40), and IL-2 is known to induce the release of IFN- $\gamma$ (41). Therefore, we hypothesized that IL-2 may increase the expression of PD-L1 by mediating the effect of IFN- $\gamma$; however, the details and mechanisms by which IL-2 
and IFN- $\alpha$ may increase expression of PD-L1 remain unclear. Based on evidence from previous studies, in combination with the present RT-qPCR data, it was concluded that OK-432 stimulated the production of a variety of cytokines, including IL-2, IFN- $\alpha$ and IFN- $\gamma$, which not only perform key roles in enhancing immunity, but increase the expression of PD-L1, thereby reducing the antitumor effect.

The antitumor immune response may be reduced by increased expression of PD-L1 through the inhibition or shielding of certain antigen-presenting cells (APCs) that are involved in antigen delivery. Together, these mechanisms may explain why treatment outcome is poor in PD-L1-positive patients with NMIBC when OK-432 is administered as a bladder intravesical agent. As treatment progresses, expression of PD-L1 increases, which directly inhibits the host antitumor immune system and T cells and may shield associated APCs, thereby promoting resistance against the antitumor actions of OK-432 in the treatment of NMIBC.

The interaction between bladder cancer cells and the immune system is a complex and dynamic process, therefore additional in vitro and in vivo mechanistic studies are required to fully elucidate the role of PD-L1 in bladder cancer and its effect on immunotherapy. However, the present study demonstrated that abnormal expression of PD-L1 is associated with recurrence and progression in bladder cancer and that it may compromise the antitumor effect of intravesical immunotherapy.

The expression of the negative costimulatory molecule PD-L1 is negatively-associated with intravesical immunotherapeutic outcome in patients with NMIBC. It was hypothesized that the mechanism involves upregulation of PD-L1 mediated by specific cytokines, thereby compromising the immune response, ultimately leading to tumor recurrence and progression. The present findings may contribute to future improvements in the efficacy of adjuvant intravesical therapy in patients with NMIBC according to the expression of PD-L1.

\section{References}

1. Irani J, Bernardini S, Bonnal JL, Chauvet B, Colombel M, Davin JL,Laurent G, Lebret T, Maidenberg M, Mazerolles C, et al: Urothelial tumors. Prog Urol 17: 1065-1098, 2007 (In French).

2. Milojevic B, Dzamic Z, Kajmakovic B, Milenkovic Petronic D and Sipetic Grujicic S: Urothelial carcinoma: Recurrence and risk factors. J BUON 20: 391-398, 2015.

3. Kaufman DS, Shipley WU and Feldman AS: Bladder cancer. Lancet 374: 239-249, 2009.

4. Eifler JB, Scarpato KR and Clark PE: Management of noninvasive bladder cancers. Curr Opin Oncol 27: 185-190, 2015.

5. Seliger B and Quandt D: The expression, function, and clinical relevance of B7 family members in cancer. Cancer Immunol Immunother 61: 1327-1341, 2012.

6. Shi L, Chen SH, Yang LJ and Li Y: The role of PD-1 and PD-L1 in T-cell immune suppression in patients with hematological malignancies. J Hematol Oncol 6: 74, 2013.

7. Wada H, Isobe M, Kakimi K, Mizote Y, Eikawa S, Sato E, Takigawa N, Kiura K, Tsuji K, Iwatsuki K, et al: Vaccination with NY-ESO-1 overlapping peptides mixed with Picibanil OK-432 and montanide ISA-51 in patients with cancers expressing the NY-ESO-1 antigen. J Immunother 37: 84-92, 2014.

8. Tano T, Okamoto M, Kan S, Bando T, Goda H, Nakashiro K, Shimodaira S, Koido S, Homma S, Fujita T, et al: Immunochemoradiotherapy for patients with oral squamous cell carcinoma: Augmentation of OK-432-induced helper T cell 1 response by 5-FU and X-ray irradiation. Neoplasia 15: 805-814, 2013.
9. Nakamoto Y, Mizukoshi E, Kitahara M, Arihara F, Sakai Y, Kakinoki K, Fujita Y, Marukawa Y, Arai K, Yamashita T, et al: Prolonged recurrence-free survival following OK432-stimulated dendritic cell transfer into hepatocellular carcinoma during transarterial embolization. Clin Exp Immunol 163: 165-177, 2011.

10. Yoo C, Do HA, Jeong IG, Park H, Hwang JJ, Hong JH, Cho JS, Choo MS, Ahn H and Kim CS: Efficacy of dendritic cells matured early with OK-432 (Picibanil), prostaglandin E2, and interferon-alpha as a vaccine for a hormone refractory prostate cancer cell line. J Korean Med Sci 25: 1284-1290, 2010.

11. Fujimoto T, Duda RB, Szilvasi A, Chen X, Mai M and O'Donnell MA: Streptococcal preparation OK-432 is a potent inducer of IL-12 and a T helper cell 1 dominant state. J Immunol 158: 5619-5626, 1997.

12. Saito M, Ebina T, Koi M, Yamaguchi T, Kawade Y and Ishida N: Induction of interferon-gamma in mouse spleen cells by OK-432, a preparation of Streptococcus pyogenes. Cell Immunol 68: 187-192, 1982.

13. Livak KJ and Schmittgen TD: Analysis of relative gene expression data using real-time quantitative PCR and the 2(-Delta Delta C(T)) method. Methods 25: 402-408, 2001.

14. Sylvester RJ, van der Meijden APM, Oosterlinck W, Witjes JA, Bouffioux C, Denis L, Newling DW and Kurth K: Predicting recurrence and progression in individual patients with stage Ta T1 bladder cancer using EORTC risk tables: A combined analysis of 2596 patients from seven EORTC trials. Eur Urol 49: 466-477, 2006.

15. Alexandroff AB, Nicholson S, Patel PM and Jackson AM: Recent advances in bacillus Calmette-Guerin immunotherapy in bladder cancer. Immunotherapy 2: 551-560, 2010.

16. Gandhi NM, Morales A and Lamm DL: Bacillus Calmette-Guérin immunotherapy for genitourinary cancer. BJU Int 112: 288-297, 2013.

17. Babjuk M, Oosterlinck W, Sylvester R, Kaasinen E, Böhle A and Palou-Redorta J; European Association of Urology (EAU): EAU guidelines on non-muscle-invasive urothelial carcinoma of the bladder. Eur Urol 54: 303-314, 2008.

18. Tian K, Han B, Shen Y, Li C and Xuan Y: Investigation on immune function and chest drainage in patients with thoracic malignancies using the streptococcal agent Sapylin. J Cancer Res Ther 10: 1030-1032, 2014.

19. Yang Y, Gao E, Liu X, Ye Z, Chen Y, Li Q, Qu J, Dai X, Wang O, Pan Y and Zhang X: Effectiveness of OK-432 (Sapylin) to reduce seroma formation after axillary lymphadenectomy for breast cancer. Ann Sur Oncol 20: 1500-1504, 2013.

20. Matsubara N, Itoh K, Mukai $\mathrm{H}$ and Nagai S: Long-term outcome of pleurodesis with OK-432 in metastatic breast cancer: A new risk model for success from an analysis of 75 cases. Int J Clin Oncol 17: 470-476, 2012.

21. Liu J, Liu X, Cui F, Chen G, Guan Y and He J: The efficacy of the inhalation of an aerosolized Group A streptococcal preparation in the treatment of lung cancer. Chin J Cancer Res 24: 346-352, 2012.

22. Sun XZ and QIU SP: Intravesical instillation of picibanil in prophylaxis of local recurrence after resecion of bladder cancer and its mechanism. China Journal of Modern Medicine: 49-51, 54, 2004.

23. Tian YF, Tang K, Guan W, Yang T, Xu H, Zhuang QY and Ye ZQ: OK-432 suppresses proliferation and metastasis by tumor associated macrophages in bladder cancer. Asian Pac J Cancer Prev 16: 4537-4542, 2015.

24. Sudo T, Aruga A, Shimizu K, Matsushita N and Takasaki K: OK432-activated natural killer cells enhanced trastuzumab (Herceptin (R)-mediated antibody-dependent cellular cytotoxicity in patients with advanced cancer. Anticancer Res 26: 4327-4333, 2006.

25. Okamoto M, Ohe G, Furuichi S, Nishikawa H, Oshikawa T, Tano T, Ahmed SU, Yoshida H, Moriya Y, Matsubara S, et al: Enhancement of anti-tumor immunity by lipoteichoic acid-related molecule isolated from OK-432, a streptococcal agent, in athymic nude mice bearing human salivary adenocarcinoma: Role of natural killer cells. Anticancer Res 22: 3229-3239, 2002.

26. Nakayama F, Iwagaki $H$, Gouchi A, Hizuta A, Isozaki $H$, Takakura N and Tanaka N: Effect of streptococcal lyzate OK-432 on peripheral blood mononuclear cells in gastric cancer patients. J Med 29: 199-215, 1998.

27. Dong H, Zhu G, Tamada K and Chen L: B7-H1, a third member of the B7 family, co-stimulates T-cell proliferation and interleukin-10 secretion. Nat Med 5: 1365-1369, 1999. 
28. Chen C, Qu QX, Huang JA, Zhu YB, Ge Y, Wang Q and Zhang XG: Expression of programmed-death receptor ligands 1 and 2 may contribute to the poor stimulatory potential of murine immature dendritic cells. Immunobiology 212: 159-165, 2007.

29. Huang Y, Zhang SD, McCrudden $C$, Chan KW, Lin Y and Kwok HF: The prognostic significance of PD-L1 in bladder cancer. Oncol Rep 33: 3075-3084, 2015.

30. Huang Y, Zhang SD, McCrudden C, Chan KW, Lin Y and Kwok HF: The presence of programmed death 1 (PD-1)-positive tumor-infiltrating lymphocytes is associated with poor prognosis in human breast cancer. Breast Cancer Res Treat 139: 667-676, 2013.

31. Maine CJ, Aziz NH, Chatterjee J, Hayford C, Brewig N, Whilding L, George AJ and Ghaem-Maghami S: Programmed death ligand-1 over-expression correlates with malignancy and contributes to immune regulation in ovarian cancer. Cancer Immunol Immunother 63: 215-224, 2014.

32. Afreen S and Dermime S: The immunoinhibitory B7-H1 molecule as a potential target in cancer: Killing many birds with one stone. Hematol Oncol Stem Cell Ther 7: 1-17, 2014.

33. Inman BA, Sebo TJ, Frigola X, Dong H, Bergstralh EJ, Frank I, Fradet Y, Lacombe L and Kwon ED: PD-L1 (B7-H1) expression by urothelial carcinoma of bladder and BCG-induced granulomata: Associations with localized stage progression. Cancer 109: 1499-1505, 2007.

34. Boorjian SA, Sheinin Y, Crispen PL, Farmer SA, Lohse CM, Kuntz SM, Leibovich BC, Kwon ED and Frank I: T-cell coregulatory molecule expression in urothelial cell carcinoma: Clinicopathologic correlations and association with survival. Clin Cancer Res 14: 4800-4808, 2008.
35. Xing L, Ping L, Bo Z, Keqin Z, Fengshuo J and Yanfeng L: Phenotype and metergasis of dendritic cells from peripheral blood of bladder carcinoma patients. J Third Mil Med Univ: 901-904, 2013.

36. Dong HD, Strome SE, Salomao DR, Tamura H, Hirano F, Flies DB, Roche PC, Lu J, Zhu G, Tamada K, et al: Tumor-associated B7-H1 promotes T-cell apoptosis: A potential mechanism of immune evasion. Nat Med 8: 793-800, 2002.

37. Keir ME, Butte MJ, Freeman GJ and Sharpe AH: PD-1 and its ligands in tolerance and immunity. Annu Rev Immunol 26: 677-704, 2008.

38. Dunn GP, Koebel CM and Schreiber RD: Interferons, immunity and cancer immunoediting. Nat Rev Immunol 6: 836-848, 2006.

39. Liu JZ, Hamrouni A, Wolowiec D, Coiteux V, Kuliczkowski K, Hetuin D, Saudemont A and Quesnel B: Plasma cells from multiple myeloma patients express B7-H1 (PD-L1) and increase expression after stimulation with IFN-\{gamma\} and TLR ligands via a MyD88-, TRAF6-, and MEK-dependent pathway. Blood 110: 296-304, 2007.

40. Parsa AT, Waldron JS, Panner A, Crane CA, Parney IF, Barry JJ, Cachola KE, Murray JC, Tihan T, Jensen MC, et al: Loss of tumor suppressor PTEN function increases B7-H1 expression and immunoresistance in glioma. Nat Med 13: 84-88, 2007.

41. Camisaschi C, Filipazzi P, Tazzari M, Casati C, Beretta V, Pilla L, Patuzzo R, Maurichi A, Cova A, Maio M, et al: Effects of cyclophosphamide and IL-2 on regulatory CD4+ T cell frequency and function in melanoma patients vaccinated with HLA-class I peptides: Impact on the antigen-specific T cell response. Cancer Immunol Immunother 62: 897-908, 2013. 